\title{
Features and functional importance of key residues of the Mycobacterium tuberculosis cytochrome bd oxidase
}

\author{
Ekaterina Sviriaeva $^{1,2 \#}$, Malathy Sony Subramanian Manimekalai ${ }^{1, \#}$, Gerhard Grüber ${ }^{1, *}$ and Kevin \\ Pethe $^{1,2, *}$
}

From the ${ }^{1}$ Nanyang Technological University, School of Biological Sciences, 60 Nanyang Drive, Singapore 637551, Republic of Singapore, ${ }^{2}$ Lee Kong Chian School of Medicine, 59 Nanyang Drive, Singapore 636921, Republic of Singapore

${ }^{\#}$ These authors have equal contribution

* To whom correspondence may be addressed: Prof. Dr. Gerhard Grüber, Tel.: + 65 - 6316 2989, E-mail: ggrueber@ntu.edu.sg and Prof. Dr. Kevin Pethe, Tel.: + 65 - 6592 3958, E-mail: kevin.pethe@ntu.edu.sg 
Cytochrome $b d$ (cyt- $b d$ ) oxygen reductases have a high affinity to oxygen and use the two electrons provided by ubiquinol or menaquinol - like in mycobacteria - to reduce oxygen to water. Although they do not pump protons from the cytoplasmic to the periplasmic side, they generate a proton motive force due to the release of protons after quinol oxidation. Here we show that the mycobacterial cyt- $b d$ has a number of specific features including a 17-residue stretch (307SGVTLQGIRDLQQEYQQ 323 ) near the Q-loop of the M. tuberculosis subunit CydA and a ${ }_{412} \mathrm{QLVRLTVKA}_{420}$ region on the periplasmic side. Site directed mutagenesis and whole-bacteria assays demonstrated that these mycobacteria-specific stretches are essential for the oxidase's function. Single amino acid substitutions around the ${ }_{307}$ SGVTLQGIRDLQQEYQQ 323 stretch revealed the importance of the aromatic residue Y330 in oxygen consumption and consequently in ATP synthesis. A moderate reduction and no effect was observed for mutants F325 and Y321, respectively, while the double mutant CydAY321/F325 drastically reduced enzyme activity. In addition, single mutants of the mycobacterial cyt- $b d$ were generated to probe the role of proposed critical residues for proton shuffling. Further data demonstrate that amino acids W64 and F18 in the CydB subunit might be important as any slight destabilization of the hydrophobic environment near them makes the enzyme inactive. Finally, the potential of the mycobacterial cyt- $b d$ as a drug target is discussed.

Keywords: bioenergetics, respiration, proton transfer, Mycobacterium tuberculosis, oxygen, cytochrome oxidases

Mycobacterium tuberculosis is an obligate aerobe that is strictly dependent on oxygen to meet its energetics demand during growth. Without any effective fermentative process, the oxidative phosphorylation (OXPHOS) pathway is crucial to maintain redox homeostasis and synthesis of sufficient amount of ATP. ${ }^{1-2}$ Most mycobacteria possess two terminal oxidases: a proton-pumping cytochrome $b c c: a a_{3}$ oxidase supercomplex (cyt-bcc:aan) and a non-proton pumping, less energetically efficient, cytochrome $b d$ oxidase (cyt- $b d) .{ }^{3}$ The discovery of several small-molecules targeting the cyt- $b c c: a a_{3}$ validated this respiratory branch as a target for drug development for the treatment of tuberculosis, Buruli ulcer and potentially leprosy. ${ }^{4-10}$ Furthermore, the synthetic lethality between the cyt-bcc:aa3 and the cyt- $b d$ highlights the promise of targeting the $b d$-type terminal oxidase to rapidly eradicate $M$. tuberculosis infections. ${ }^{11-12}$ The value of the cyt-bd as a drug target is further reinforced by the observation that deletion of the cyt- $b d$-encoding genes sensitizes mycobacteria to bedaquiline and clofazimine, both known for their interference on the OXPHOS pathway. ${ }^{13-15}$ Therefore, specific drug-mediated inhibition of the cyt- $b d$ could increase efficacy and possibly decrease treatment duration.

The $M$. tuberculosis cyt- $b d$ is encoded by the $c y d A B D C$ operon; the $c y d A B$ genes encode the catalytic subunits $\mathrm{Cyd} A$ and $\mathrm{CydB}$, respectively, while the $c y d D C$ genes encode a putative cytochrome synthesis ABC-transporter whose function may be required for the proper assembly of the complex. ${ }^{16}$ The mycobacterial cyt- $b d$ is poorly characterized and its putative functions are only inferred from those of its homologues in Escherichia coli and other species. ${ }^{17-22}$ The crystal 
structure of the Geobacillus thermodenitrificans cyt- $b d$ gave a first insight into the structure and function of a $b d$-type terminal oxidase (PDB ID: 5DOQ), while the newly published structure of the complex from Escherichia coli (PDB ID: 6RKO) offers further understanding into this class of terminal oxidase. ${ }^{23-24}$ The cyt- $b d$ consists of two similar subunits and each unit has nine transmembrane helixes with two bundles of four helices and one peripheral helix. ${ }^{23-24}$ Subunit CydA contains the water-exposed quinol-binding site called the Q-loop and three hemes: a lowspin heme $b_{558}$, a high-spin heme $b_{595}$ and a chlorin-type heme $d$. It is proposed that heme $\mathrm{b}_{558}$ receives the electrons from the process of quinol oxidation in the Q-loop and passes the electrons directly to the next heme through a conserved tryptophan residue (W374 in G. thermodenitrificans and W441 in E. coli) ${ }^{23-24}$. Interestingly, the hemes' configuration and their electrochemical properties differ between the two species, implying distinct electron transfer pathways. Indeed, the heme $d$ and heme $b_{595}$ are likely required for the simultaneous transfer of 4 electrons onto a bound oxygen molecule, resulting in the breakage of its dioxygen bond in $G$. thermodenitrificans ${ }^{23}$, while a sequential electron transfer from heme $b_{558}$ via heme $\mathrm{b}_{595}$ to heme $d$ was proposed for the $E$. coli cyt- $b d$. The oxygen entry sites are also distinct: in E. coli the subunit CydH seals the hydrophobic cleft that serves as the oxygen entry site in the G. thermodenitrificans enzyme, and instead relies on an O-channel for oxygen diffusion to the reaction center. ${ }^{23-24}$ Protons for oxygen reduction can be donated from the cytosol by a hydrophilic $\mathrm{H}$-channel with two proposed routes on the surface of each of the subunits (CydA- and CydB pathway) in both structures. ${ }^{23-24}$ However, so far these pathways have not been functionally proven through mutation studies. Furthermore, the identification and characterization of possible proton pathways within the mycobacterial cyt- $b d$ would be an important step towards the development of specific inhibitors.

In this manuscript, we performed a comparative analysis of the $M$. tuberculosis cyt- $b d$ with E. coli and G. thermodenitrificans, and identified regions and amino acid residues unique to mycobacteria. The importance of the selected regions and amino acid residues on the enzymatic function was then elucidated by targeted mutation and functional assays. The mapping of functionally important residues represents a first step toward structure-based drug design for the development of potent cyt- $b d$ inhibitors.

\section{RESULTS}

Identification of unique features in mycobacterial cyt-bd-We compared sequences of cyt-bd subunits CydA and CydB across the mycobacteria, including M. tuberculosis, M. smegmatis, and M. bovis BCG, together with E. coli, G. thermodenitrificans and other bacteria (Fig. S1, S2). Of note, the cyt-bd of M. tuberculosis and $M$. bovis BCG are $100 \%$ identical. This multiple amino acid sequence alignment revealed two mycobacteria-specific regions in the subunit CydA. Firstly, we observed an insertion near the C-terminus region of nine residues ${ }_{412} \mathrm{QLVRLTVKA}_{420}$ (numbering according to M. tuberculosis sequence) in the mycobacterial subunit CydA (Fig. S1). Secondly, a unique region spanning 17 residues (307SGVTLQGIRDLQQEYQQ ${ }_{323}$ ) was identified in the mycobacterial subunit CydA (Fig. S1). Interestingly, this insertion corresponds to a 55residue stretch from D301 to I372 found in E. coli at a similar position predicted to be near the 
quinol binding region. ${ }^{25}$ In comparison, the $G$. thermodenitrificans cyt-bd amino acid sequence reveals a stretch of thirteen residues at this position (299AAVVTGLNDTPED ${ }_{311}$ ) with no homology to the mycobacterial or E. coli counterparts. Moreover, the length of the insertion varies among different organisms. In the case of subunit $\mathrm{CydB}$, the $E$. coli subunit has a long insertion of around 33 residues near from T219 to Q220 (according to M. tuberculosis sequence), which is absent in both mycobacteria and G. thermodenitrificans (Fig. S2). The significance of this longer loop in E. coli is not yet established.

Construction of a three-dimensional model of the M. tuberculosis cyt-bd-The 3D model of the M. tuberculosis cyt- $b d$ was generated by homology modelling using the $G$. thermodenitrificans crystal structure (PDB ID: 5DOQ), which was the only template available at the start of the project. $^{23}$ The $M$. tuberculosis cyt- $b d$ heme groups were also modelled based on the G. thermodenitrificans structure. A model with a sequence coverage of around $90 \%$ and a GMQE of approximately 0.61 was predicted. The model compared well with the G. thermodenitrificans structure with an r.m.s. deviation of $0.27 \AA$ (Fig. 1B). It also superimposed with the recently solved cryo-EM structure of E. coli (PDB ID: 6RKO) with an r.m.s.d. of $2.27 \AA$ (Fig. 1C).

The $M$. tuberculosis cyt- $b d$ model presented in figure 1A shares a similar topology with the $G$. thermodenitrificans structural template. The extra 17-residue stretch (307SGVTLQGIRDLQQEYQQ ${ }_{323}$ ) near the Q-loop of the M. tuberculosis subunit CydA model is located on the periplasmic side of the model and a short helix is formed by residues I314 to E320 (Fig. 1A). In comparison, the corresponding region in the G. thermodenitrificans crystal structure (299AAVVTGLN $\mathrm{A}_{306}$ ) is shorter and forms a loop, while in E. coli it was shown to interact with an aurachin D-type inhibitor. ${ }^{23-24}$ Next, the mycobacteria-specific short region ${ }_{412}$ QLVRLTVKA $_{420}$ lies on the periplasmic side in the model and contains a predicted short helical region from V414 to Q420 (Fig. 1A). In order to decipher the importance of these two unique regions, the two deletion mutants $\mathrm{Cyd}_{\triangle 307-323}$ and $\mathrm{CydA}_{\triangle 412-420}$ were constructed (see below). In addition, a mutant $\mathrm{CydA}_{\triangle 318-323}$ with a shorter deletion was created to better understand the interactions around the first putative mycobacteria-specific helical region.

The positions of cysteine and histidine residues, which play a critical role in heme-containing and electron-transferring proteins, were identified in subunit CydA of the M. tuberculosis cyt- $b d$ model. As shown in figure 2A, the two mycobacteria-specific cysteine C266 and C285 are located on the periplasmic side of the model. Interestingly, C266 is in close proximity (approximately within $5 \AA$ ) to the menaquinol-loop, implying a role in menaquinol binding and oxidation. In addition, the two cysteines are also near each other. Even though a predicted distance of about 7.4 $\AA$ between the sulphur atoms render them unsuitable to form a disulphide bond in the model, this does not exclude the possibility that the two sulphur atoms of C266 and C285 come in closer vicinity to form a bond during the catalytic steps of menaquinol-binding and oxidation, which was previously described for the redox-modulation of the chloroplast F-ATP synthase. ${ }^{26}$ The third mycobacteria-specific cysteine residue $\mathrm{C} 230$ is near the cytoplasmic side. Of the two histidine residues $\mathrm{H} 246$ and $\mathrm{H} 213$, amino acid $\mathrm{H} 246$ is close to the heme group with a distance of about 7.4 $\AA$, while H213 lies on the cytoplasmic side (Fig. 2A). 
In subunit CydA, the conserved and non-conserved residues within the mycobacterium species were identified based on sequence alignment (Fig. S1) and were mapped onto the M. tuberculosis cyt-bd model (Fig. 2B). The mapping revealed that the residue variations amongst the mycobacterium species lie on four helices 10-13 (Fig. 2B). The residual variations are spread across the helices from the periplasmic to the cytoplasmic side without being particularly concentrated on any one region. However, significant non-conservancy between the mycobacterium species and other species was noted near the quinol binding region, the unique mycobacteria-specific region $\left({ }_{412} \mathrm{QLVRLTVKA}_{420}\right)$ and the extra stretch (307SGVTLQGIRDLQQEYQQ 323 ) near the Q-loop. Analysis of aromatic residues in this nonconserved region showed that a cluster of three aromatic residues comprising Y321, Y330 and F325 in $M$. tuberculosis, lies in between the unique mycobacterium extension region (412QLVRLTVKA 420 ) and stretch ${ }_{307}$ SGVTLQGIRDLQQEYQQ $_{323}$ in the menaquinol binding region. This aromatic cluster might make the region surrounding it hydrophobic on the periplasmic side. Although such aromatic clusters can also be found in other species (Fig. S1), this cluster is of possible interest. It is predicted to be closer to the mycobacteria-specific region $\left({ }_{412} \mathrm{QLVRLTVKA}_{420}\right)$ and to the extra stretch $\left.{ }_{307} \mathrm{SGVTLQGIRDLQQEYQQ}_{323}\right)$ in the 3D model of $M$. tuberculosis cyt- $b d$, thus possibly contributing to the Q-loop stability. In addition, the F252 may in part become relevant for stabilizing menaquinol-binding, while two other residues, F242 and F394, may affect the porphyrin-ring of heme $b$ (Fig. 2B). In the binding of BDQ, the large number of aromatic residues within the turbine subunit $c$ of the mycobacterial F-ATP synthase provides an ideal epitope for BDQ not only to bind but also to interact specifically with the mycobacterial protein. ${ }^{27}$ In this context, the mycobacteria-specific aromatic residues of subunit CydA might provide a good target for structure-based drug design. To understand the relevance of this aromatic cluster, three single mutants $\mathrm{CydA}_{\mathrm{Y} 321 \mathrm{~A}}, \mathrm{CydA}_{\mathrm{F} 325 \mathrm{~A}}$, and $\mathrm{CydA}_{\mathrm{Y} 330 \mathrm{~A}}$ and a double mutant CydA $1321 \mathrm{~A} / \mathrm{F} 325 \mathrm{~A}$ were created and studied (see below).

The cyt- $b d$ sequence is $100 \%$ homologous between $M$. tuberculosis and M. bovis BCG, however, some residual differences were identified when compared with the M. smegmatis sequence (marked in yellow in Fig. 2B). Particularly, three regions showed distinctive differences between $M$. tuberculosis and M. smegmatis: ${ }_{267} \mathrm{DTQT}_{270}{ }_{237} \mathrm{TAG}_{239}$, and ${ }_{210} \mathrm{STTHADSDTQA}_{220}$. The region ${ }_{267} \mathrm{DTQT}_{270}$ is near the Q-loop region while ${ }_{237} \mathrm{TAG}_{239}$ lies in the middle of helix 10 of subunit CydA. The region ${ }_{210} \mathrm{STTHADSDTQA}_{220}$ is situated in the loop region below the helix 10 of subunit CydA. The ${ }_{237} \mathrm{TAG}_{239}$ region is close to heme $b_{595}$, with the residue T237 having the shortest distance to heme group (4.6 $\AA$ ). However, the significance of these regions is yet to be deciphered.

Identification of residues required for the proton transfer pathway in subunit CydB-Two potential proton transfer pathways (CydA- and CydB pathways) were predicted in the crystal structure of the G. thermodenitrificans cyt- $b d$ (PDB ID: 5DOQ), which passes protons from the cytoplasm to the high-spin heme $b_{595 .}{ }^{23}$ In G. thermodenitrificans the CydB pathway is predicted to be formed by key residues H36, S32, Y103, D25, R99 and Y18, belonging to helices 1-4 of subunit CydB, as well as amino acid $\mathrm{T} 73$ from subunit CydA. ${ }^{23}$ The corresponding residues in the 
M. tuberculosis cyt-bd model are H37, M32, I105, D25, R101 and F18 of subunit CydB, and I70 of subunit CydA, respectively (Fig. 3). In order to understand the functional significance of this region, mutations were designed around the predicted $\mathrm{CydB}$ pathway, including the mutant $\mathrm{CydB}_{\mathrm{L} 21 \mathrm{~A}}$ in helix 1 of subunit CydB since this residue lies between two predicted critical residues F18 and D25 in the 3D model. The structure of E. coli revealed that the H-channel and O-channel are located close to each other. Interestingly, the totally conserved W63 residue (W64 in M. tuberculosis) is located near the point of convergence of the two channels. ${ }^{24}$ Also, W64 shares a distance of $4.4 \AA$ with F18 in the M. tuberculosis model, indicating possible stacking interactions between them, hence the mutant $\mathrm{CydB}_{\mathrm{w} 64 \mathrm{~A}}$ was created. Moreover, T53 in helix 2 of subunit $\mathrm{CydB}$ was a residue of interest because of its proximity to the possible entrance of the predicted CydB pathway. T53 is also positioned close to amino acid N52, which might interact with residue F115 of subunit $\mathrm{CydA}$ and stabilize cyt- $b d$, thus the mutant $\mathrm{CydB}_{\mathrm{T} 53 \mathrm{~A}}$ was genetically engineered. Next, as shown in the model on figure 3, the amino acids F311, L314 and V316 in helix 11 of subunit CydB might lie on the interface between subunits CydA and CydB. Specifically, V316 might interact with T108 of subunit CydA, while F311 and L314 might be involved in hydrophobic interactions with L100 and F105 of subunit CydA, respectively. Therefore, the single mutants $\mathrm{CydB}_{\mathrm{F} 311 \mathrm{~A}}, \mathrm{CydB}_{\mathrm{L} 314 \mathrm{~A}}$, and $\mathrm{CydB}_{\mathrm{V} 316 \mathrm{~A}}$ were also created to better understand the functional significance of these residues.

Deletion of the unique helical regions of the $M$. tuberculosis cyt-bd abolished enzymatic activity-The genetically engineered subunit CydA mutants $\mathrm{CydA}_{\triangle 307-323}$, Cyd $\mathrm{A}_{\Delta 318-323}$ and $\mathrm{CydA}_{\triangle 412-420}$, which lack the unique helical regions of mycobacterial cyt- $b d$, were tested in functional assays in $M$. bovis BCG $\triangle c y d A B .{ }^{11}$ The knockout strain was complemented with an integrative plasmid expressing either parental or a mutated form of the cydABDC operon. Complemented strains were treated with Q203 to inhibit the cyt- $b c c: a a_{3}$ respiratory branch. Under these experimental conditions, the bacteria are completely dependent on a functional cyt- $b d$ to maintain ATP levels and oxygen consumption, two parameters that can be quantified in whole bacteria. ATP production was shown to be significantly restored in the strain complemented with parental $c y d A B D C$ operon in presence of Q203 (Fig. S3A). The OCR was measured with 2 methods: quantitative MitoXpress ${ }^{\circledR}$ probe (Fig. S3, C-E) and qualitative methylene blue (Fig. S3F). The ATP level and MitoXpress ${ }^{\circledR}$ data are further presented as a ratio of Q203-treated to drug-free control for easier interpretation (Fig. S3B, E).

We found that the mutants $\mathrm{CydA}_{\triangle 318-323}, \mathrm{CydA}_{\triangle 307-323}$, and $\mathrm{CydA}_{\triangle 412-420}$ were inactive, as witnessed by the inability of the recombinant strains to restore ATP levels (Fig. 4A, Fig. S4). OCR ratio measurement confirmed the inactivity of the cyt- $b d$ variants bearing deletion of the two mycobacteria-specific helical regions (Fig. 4B). The result was finally supported by qualitative methylene blue-based assay (Fig. 4C). These phenotypic observations give a clear indication that these regions are essential for cyt- $b d$ function. In addition, since the mutants $\mathrm{CydA}_{\triangle 318-323}$ and $\mathrm{CydA}_{\triangle 307-323}$ revealed the same effects, the data identified the ${ }_{318} \mathrm{QQEYQQ}_{323}$ stretch as the more critical residues in the ${ }_{307}$ SGVTLQGIRDLQQEYQQ $_{323}$ stretch. Taken together, the results indicate the importance of the mycobacterial Q-loop for the complex assembly. 
Substitution of single amino acids reveals residues essential for cyt-bd function-The consequences of the CydAy321A, CydA $\mathrm{F}_{\mathrm{F} 25 \mathrm{~A}}$, and CydAy330A mutations on the cyt- $b d$ were evaluated. The substitution Y321A had no consequence on enzymatic activity and the F325A substitution resulted in $25-60 \%$ reduction of the OCR and ATP ratios, while the single substitution of Y330A resulted in a near ablation of the cyt- $b d$ function (Fig. 5). This observation is consistent with the predictions from the cyt- $b d$ model: Y330 and F325 are located deeper in the model and closer to the predicted menaquinol binding site, thus likely to be more important, while Y321 is positioned more to the surface of cyt- $b d$. Moreover, substituting the three amino acids with a smaller amino acid such as alanine might interfere with the local protein arrangement and increase the flexibility of the loop region, possibly contributing to the observed effect. Thus, F325 and Y330 could be more important for stabilizing in this structural region of the oxidase. Interestingly, the combination of two substitutions Y321A and F325A resulted in almost complete reduction of oxidase activity (Fig. 5).

The predicted proton shuffling area is crucial for cyt-bd activity-Next, we focused on the function of the predicted proton shuffling area. Upon substitution of selected amino acids to neutral alanine in the subunit CydB, we observed varied effects. Substitution of L314A and V316A did not affect cyt- $b d$ activity (Fig. 6). This finding suggests that amino acids L314 and V316 of CydB do not play a critical role in proton shuffling to the oxygen reaction centre (Fig. 6). Substitutions of L21A, T53A, and F311A had more pronounced effects on the activity of cyt- $b d$, resulting in $20-50 \%$ reduction in ATP and OCR ratios (Fig. 6). The most striking effect was caused by the $\mathrm{CydB}_{\mathrm{W} 64 \mathrm{~A}}$ substitution that abrogated enzymatic efficiency completely (Fig. 6). This might signify that the disruption of interactions in the complex near the totally conserved tryptophan residue interferes with the proton shuffling or oxygen diffusion to the oxygen reaction centre and causes significant reduction in activity.

The residue L21 might provide a hydrophobic environment near the proton transfer pathway along with W64 and F18. This micro-hydrophobic environment could stabilize the channel for proper proton flow. Substitution of L21 and W64 to alanine would have reduced the hydrophobicity in the surroundings, thereby causing a slight destabilization in the channel, which resulted in the reduced activity. F311 is found at the interface of the complex which has hydrophobic interaction with L100 of subunit CydA. When this is substituted to alanine, a smaller and less hydrophobic residue, the hydrophobic environment in the interface was possibly perturbed, weakening the complex and leading to lower activity. In order to substantiate the above claim, a more drastic substitution to a charged residue was attempted. L21 and L314 were substituted by arginine, while F311 was substituted by serine; the substitutions were chosen to keep the helical penalties similar. Interestingly, these substitutions resulted in a dramatic inhibition of cyt-bd activity (Fig. 7). These observations suggest the importance of a hydrophobic environment for the complex formation and proper function of the subunit CydB proton shuffling pathway.

Cyt-bd integrity is severely affected by deletions, but not by amino acid substitutions-To determine whether the introduction of these mutations had an effect on complex assembly, we 
analysed the heme contents on purified plasma membranes from the parental and five mutant strains. Previously, the spectral properties of cyt- $b d$ were reported by Theßeling et al. They noted two significant signature peaks: at $563 \mathrm{~nm}$ for heme $b$ and at $630 \mathrm{~nm}$ for heme $d \cdot{ }^{28-29}$ Since heme $b$ is present in the cyt-bcc:aa3 complex and thus is not specific to cyt- $b d$, we focused on the specific heme $d$ peak. For the study, the heme content was analysed in purified bacterial membranes as previously described, with modifications. ${ }^{17,30}$ The membranes were isolated from all of the strains under the same conditions. The peak at $630 \mathrm{~nm}$ could not be detected in the M. bovis BCG parental strain, or from the recombinant strain expressing $c y d A B D C$ from an integrative vector (Fig. S5A), which is likely due to a low level of intrinsic expression of the complex. ${ }^{17}$ Of note, no effect on proton translocation in the membranes was detected upon deletion of cyt- $b d$ (Fig. S5B) reflecting the missing vectorial proton transfer of cyt- $b d$ from the cytoplasm to the intermembrane space. Moreover, these data show that possible differences of cyt- $b d$ mutants in proton translocation may not be detectable using classical proton-pumping experiments.

To increase the expression of the cyt- $b d$, we transferred sequences of the parental, $\mathrm{CydA}_{\triangle 318-}$ 323, $\mathrm{CydA}_{\triangle 412-420}, \mathrm{CydA}_{\mathrm{Y} 330 \mathrm{~A}}, \mathrm{CydB}_{\mathrm{L} 314 \mathrm{~A}}$, and $\mathrm{CydB}_{\mathrm{W} 64 \mathrm{~A}}$ mutants to a high-copy number vector pMV262. We first confirmed that the mutants expressed from the high-copy number vector were still non-functional (Fig. S6). Interestingly, $\mathrm{CydA}_{\mathrm{Y} 330 \mathrm{~A}}$ and $\mathrm{CydB}_{\mathrm{W} 64 \mathrm{~A}}$ mutants did not show complete depletion of ATP upon Q203 treatment, which was in contrast to the outcome observed when the mutations were transformed in a single-copy plasmid (Fig. S5A). This suggests that the these mutations lead to a severe but not a complete loss of function. The difference between severeand complete-loss-of-function phenotypes were previously indistinguishable, most likely due to the limitations of the assay's detection sensitivity. However, overexpression of the mutant proteins allowed for the amplification of the phenotypic signal (ATP rescue) deriving from even partial enzyme efficiency, making impaired enzymatic function detectable in the same assay. As for the deletion mutants, the lack of phenotypic complementation even in the strain carrying high gene copy numbers confirmed total loss of function and further highlighted the importance of the specific loops for proper assembly of the protein. The heme $d$-specific peak at $630 \mathrm{~nm}$ wavelength was detected at a comparable level in the parental and $\mathrm{CydB}_{\mathrm{L} 314 \mathrm{~A}}$ purified membranes (Fig. 8E), while the same peak has a noticeably lower intensity in $\mathrm{CydA}_{\triangle 318-323}, \mathrm{CydA}_{\triangle 412-420}, \mathrm{CydA}_{Y 330 \mathrm{~A}}$ and $\mathrm{CydB}_{\mathrm{W} 64 \mathrm{~A}}$ mutants (Fig. 8, A-D). This suggests that the deletion of the specific regions and disturbance in amino acid sequence in cyt- $b d$ resulted in a destabilization of protein folding and assembly on the membrane, or cofactor binding to the complex, thus causing the absence of heme $d$-specific peak. Therefore, taken together with ATP production, we postulate that the deletion of the two specific helical regions in mycobacterial cyt- $b d$ severely disturbs the folding and stability of the complex. Conversely, amino acid substitutions affect cyt- $b d$ activity mostly by disturbing key molecular interactions, leading to a partial loss of function.

\section{DISCUSSION}


The emergence of multi- and extensively- drug resistant (MDR and XDR) M. tuberculosis strains is urging further search of new antituberculars to control the spread of tuberculosis. Nevertheless, since the approval of rifampin in 1963, no new antibiotic classes were approved for almost 40 years until the FDA approved bedaquiline (BDQ, Sirturo ${ }^{\circledR}$ ) for clinical use in 2012. ${ }^{31-33}$ The current treatment regimen for MDR TB lasts 12 to 24 months. This long treatment time, alongside non-compliance among patients and low efficacy remain concerning issues. Thus, there is an urgent need to develop drugs with new modes of action that would reduce treatment duration.

Mycobacteria require the OXPHOS pathway and an energized membrane to replicate and survive in a dormant state. ${ }^{1}$ In addition to the $\mathrm{F}_{1} \mathrm{~F}_{\mathrm{O}}$ ATP synthase - the target of BDQ - several other components of the OXPHOS pathway are attractive for drug development. ${ }^{3}$ For instance, the terminal oxidases are of particular interest. While the proton-pumping cyt- $b c c: a a_{3}$ attracted most of the attention thus far, the alternate $b d$-type oxidase recently emerged as a relevant target. ${ }^{11,15} \mathrm{~A}$ better understanding of the relationship between the enzyme structure and its activity is needed to initiate rational drug discovery programs.

So far only deletion studies of either the single cydA gene or the cydAB genes in mycobacteria have been reported. ${ }^{11,14}$ In this study, the biological significance of short Q-loop and possible proton shuffling area of the mycobacterial cyt- $b d$ was confirmed for the first time by using sitedirected mutagenesis and whole-bacteria assays. Such approach is attractive as the complex is in its native condition and thus artefacts of complex isolation do not affect the results.

The homology model of $M$. tuberculosis cyt- $b d$ and the amino acid sequence comparison presented in this manuscript identified two mycobacteria-specific regions in CydA, ${ }_{412}$ QLVRLTVKA $_{420}$ and ${ }_{307}$ SGVTLQGIRDLQQEYQQ $_{323}$, both predicted to be on the periplasmic side of the complex (Fig. 1). Additionally, an aromatic cluster of amino acids Y321, Y330 and F325 predicted to be close to Q-loop was described. The deletion mutants $\mathrm{CydA}_{\triangle 318-323}$ and $\mathrm{CydA}_{\triangle 412-420}$, and the substitution of Y330A and Y321A/F325A rendered the enzyme inactive (Fig. 4; Fig. 5). It is possible that the ${ }_{412} \mathrm{QLVRLTVKA}_{420}$ stretch evolved independently from the C-terminal domain of long Q-loop of E. coli to cover the periplasmic surface of CydA. ${ }^{24}$ All the three regions can form a cavity near the menaquinol binding region containing residues $\mathrm{K} 258$ and E263 in the 3D model. ${ }^{23}$ Moreover, the ${ }_{307}$ SGVTLQGIRDLQQEYQQ 323 stretch might lie near the Q-loop with its helical part ${ }_{314} \mathrm{IRDLQQE}_{320}$ close $(5.5 \AA)$ to the helix ${ }_{256} \mathrm{PMKMASAE}_{263}$. This helix was shown to hold the K258 and E263 residues, essential for menaquinol binding (K252 and E257 in G. thermodenitrificans). ${ }^{23}$ The stretch might be also involved in menaquinol binding as a similar region in the $E$ coli cyt- $b d$ interacts with an aurachin D-type inhibitor which is structurally similar to menaquinol. ${ }^{24}$ Hence, we speculate that the identified mycobacteria-specific regions might aid in the structural stabilization of the menaquinol binding site, thereby promoting substrate binding. The heme absorbance data further point to the importance of the mycobacteria-specific stretches in the Q-loop for proper assembly (Fig. 8A, B). However, this hypothesis remains to be verified using the $M$. tuberculosis cyt- $b d$ structure. The Q-loop region in the crystal structure of $G$. thermodenitrificans and in the cryo-EM structure of $E$. coli is highly flexible and therefore, the interpretation regarding the Q-loop mutants or its deletions has to be dealt with caution. ${ }^{23-24}$ 
Interestingly, while our manuscript was under revision it was reported that $E$. coli cyt- $b d$ requires its long Q-loop for optimal enzyme activity and substituting it with shorter homologs from $G$. thermodenitrificans or M. tuberculosis inactivates the complex..$^{28,34}$

We also investigated the role of amino acids possibly involved in proton shuffling to the reaction center. The proton shuffling pathways were proposed for access of the protons to the oxygen-binding site based on the crystal structure of $G$. thermodenitrificans cyt- $b d$, while cyt- $b d$ structure from E. coli revealed an H-channel for this purpose. ${ }^{23-24}$ We propose that F18 and its stacking interactions with surrounding residues might be important in the proton pathway, since the destabilization of the hydrophobic environment in its proximity led to the enzyme's loss of function, as observed by the L21 substitution (Fig. 6). The substitution of the conserved W64 of CydB made the enzyme totally inactive (Fig. 6, 8). The H- and O-channel of E. coli merge at the corresponding residue $\mathrm{W} 63$, proposing its essentiality in proper assembly of the cyt- $b d .^{24}$ Interestingly, amino acid E107, located along the O-channel, was previously shown to be important for function of the $E$. coli cyt- $b d$ by point mutation. ${ }^{24,35}$ The E107 residue of subunit CydA was essential for cyt- $b d$ function: it stayed protonated, suggesting it may be important for proton transfer or charge compensation during oxygen diffusion to the oxygen binding site, reinforcing the role of this region for oxygen reduction by the complex. ${ }^{35}$ Indeed, the structure of cyt- $b d$ from $E$. coli revealed that E107 interacts with heme $d$ and is located along the O-channel.

The environment on the interface of subunit CydA and CydB was probed by the substitution of residues F311, L314 and V316 to neutral alanine. Results revealed that those substitutions did not significantly impact the enzyme activity. However, substituting F311 and L314 with a polar residue resulted in cyt- $b d$ inactivation (Fig. 7). This suggests that the disruption of hydrophobic environment in the interface using a charged residue destabilizes the complex and leads to its inactivation.

In this manuscript we present data on mutational studies of cytochrome $b d$ oxidase from $M$. tuberculosis, an emerging drug target. The report on essential regions of mycobacterial cyt- $b d$ provides a basis for rational design of specific cyt- $b d$ inhibitors. The development of an inhibitor of cyt- $b d$ would add to the panel of antitubercular drugs and broaden choice of treatment. If used in a potent and rational combination with other OXPHOS inhibitors, cyt- $b d$ inhibition could even possibly shorten treatment duration.

\section{METHODS}

Amino acid sequence alignment of cyt-bd subunits-The multiple amino acid sequence alignments of subunits $\mathrm{CydA}$ and $\mathrm{CydB}$ of the cytochrome $b d$ oxidase were carried out using the online server clustal omega using mBed-like clustering guide tree and HMM profile-profile techniques. ${ }^{36}$ The alignments were performed for cyt-bd of M. tuberculosis, Mycobacterium smegmatis, Mycobacterium bovis BCG, E. coli and G. thermodenitrificans, and visualized using the program ESPript v3.0. ${ }^{37}$ The secondary structural information were extracted from the crystal structure of $G$. thermodenitrificans (PDB ID: 5DOQ) and embedded in the alignment. ${ }^{23}$ 
Homology modelling of the $M$. tuberculosis cyt- $b d-$ The $M$. tuberculosis cyt- $b d$ model was generated using homology modelling within the SWISS-MODEL server. ${ }^{38}$ The program accepts the target amino acid sequence and searches for evolutionary related protein structures against the template library SMTL using BLAST and HHblits. ${ }^{39-40}$ The templates were then ranked based on global model quality estimate (GMQE) and quaternary structure quality estimate (QSQE). A 3D model was generated automatically for the selected top-ranked templates using ProMod3 modelling engine that first transfers conserved atom coordinates defined by the target-template alignment. Afterwards, insertions/deletions were constructed using loop modelling.

Bacterial strains and growth conditions-The M. bovis BCG $\triangle c y d A B$ strain was previously described. ${ }^{11}$ The M. bovis BCG parental (WT) strain and derivatives were maintained in Middlebrook $7 \mathrm{H} 9$ broth medium supplemented with $0.2 \%$ glycerol, $0.05 \%$ Tween $80,10 \%$ ADS supplement. Hygromycin B was added as a selection marker at $80 \mu \mathrm{g} / \mathrm{ml}$ when required, while kanamycin A was used at $20 \mu \mathrm{g} / \mathrm{ml}$. For all experiments, cultures were harvested in early logarithmic phase, washed twice to remove glycerol, and adjusted to the desired cell density.

Strategy to introduce single amino acids substitution and short deletion in M. tuberculosis cyt$b d$-The integrative mycobacterial vector pMV306-cydABDC expressing the $c y d A B D C$ operon under the control of its native promoter was a kind gift from M. Berney. ${ }^{11}$ Genes $c y d D C$ were included to ensure proper expression of the cyt- $b d$ complex. Desired mutations were introduced to the vector using Q5 ${ }^{\circledR}$ Site-Directed Mutagenesis kit (New England Biolabs) with specific primers according to manufacturer's protocol. Sanger sequencing was used to confirm that obtained vectors carried only the desired mutation.

Vectors were transformed into $M$. bovis $\mathrm{BCG} \triangle c y d A B$ by electroporation and positive colonies were selected on $7 \mathrm{H} 10$ agar plates supplemented with $10 \%$ OADC and kanamycin A $(20 \mu \mathrm{g} / \mathrm{ml})$, empty pMV306 vector was transformed as a control. For each mutant variant at least two plasmid clones were tested in $M$. bovis BCG $\triangle c y d A B$. Each mutant strain was tested whether introducing the vector affected its growth. Growth was recorded over time in 7H9 media (BD) supplemented with $0.05 \%$ Tween 80 and $10 \%$ ADS supplement or $7 \mathrm{H} 9$ defined media with $10 \mathrm{mM}$ acetate as a carbon substrate for 10 days (Fig. S7 and S8).

Functional assays in whole cells-The functional assays rely on the essentiality of the cyt- $b d$ to maintain homeostatic levels of ATP and to consume oxygen when the function of the cyt-bcc: $a a_{3}$ is inhibited by Q203. ${ }^{11}$ Intracellular ATP quantification-ATP content in bacteria cultures was quantified using the BacTiter-Glo ${ }^{\mathrm{TM}}$ Microbial Cell Viability Assay (Promega). Bacteria cultures were adjusted to $\mathrm{OD}_{600}$ of 0.05 in $7 \mathrm{H} 9$ media without glycerol and dispensed into 96 -well white plates. Q203 was added to a final concentration of $100 \mathrm{nM}$ to inhibit the cyt-bcc: $a a_{3}$ respiratory branch. Intracellular ATP measurements were carried out according to the manufacturer's protocol using BioTek Cytation 3 microplate reader after 16 to 24 hours incubation at $37^{\circ} \mathrm{C}$. Oxygen Consumption Assays-Oxygen consumption in whole bacteria was measured using methylene blue assay or the MitoXpress ${ }^{\circledR}$ Xtra Oxygen Consumption Assay (Agilent) as described before. ${ }^{11}$ For the methylene blue assay, bacterial cultures were adjusted to an $\mathrm{OD}_{600}$ of 0.3 in $7 \mathrm{H} 9$ media without glycerol and preincubated for $5 \mathrm{~h}$ in $2 \mathrm{ml}$ screw-cap glass vials in the presence of Q203 at $100 \mathrm{nM}$ 
or vehicle control $(0.1 \%$ DMSO). Methylene blue was added at a concentration of $0.001 \%$. Tightly sealed tubes were then incubated in an anaerobic jar in order to prevent oxygen leak. Pictures of the tubes were taken immediately after closing the tubes and 4 to 6 days of incubation at $37^{\circ} \mathrm{C}$. For the MitoXpress ${ }^{\circledR}$ assay, the cultures were adjusted to $\mathrm{OD}_{600}$ of 0.7 and dispensed in black 96 -well plates (flat, clear bottom). $100 \mathrm{nM}$ Q203 or 1\% DMSO were subsequently added, followed by MitoXpress ${ }^{\circledR}$ oxygen probe (Agilent). Each well was then covered with a layer of high-sensitivity mineral oil to prevent oxygen diffusion. Fluorescence (Ex: $380 \mathrm{~nm}$, Em: $650 \mathrm{~nm}$ ) was recorded on a Cytation 3 (BioTek) reader over a 5 hours period. Data were processed according to manufacturer's instruction. Briefly, the raw readings of MitoXpress ${ }^{\circledR}$ probe (Fig. S3C, D) were quantified as the slope of the linear phase of the experiment as marked with vertical dashed lines, and ratio of OCR in Q203-treated and untreated sample is presented on Fig. S2E and in further experiments.

Membrane isolation, spectral analysis of heme content, and proton translocation assay-The mutants and WT cydABDC operon were transferred in the high-copy pMV262 vector using EcoRI/DraI restriction enzymes and transformed into the $M$. bovis BCG $\triangle c y d A B$ strain. The recombinant strains were grown in $1 \mathrm{~L}$ of $7 \mathrm{H} 9$ broth medium with $0.2 \%$ glycerol at $37^{\circ} \mathrm{C}$ with shaking until culture's $\mathrm{OD}_{600}$ reached $\sim 1.5$. Cells were harvested by centrifugation and resuspended in the buffer A containing $20 \mathrm{mM}$ MOPS, $100 \mathrm{mM} \mathrm{NaCl}$, complete EDTA-free protease inhibitor cocktail (Roche). Cell suspension was passed through a high pressure homogenizer Microfluidizer LM-20 (Microfluidics) three times at 15,000 psi. Homogenate was centrifuged at 15,000 g for 20 minutes to remove cell debris. The supernatant was first centrifuged at $27,000 \mathrm{~g}$ for $40 \mathrm{~min}$ and then at $150,000 \mathrm{~g}$ for 1 hour at $4{ }^{\circ} \mathrm{C}$. Final pellet was resuspended in the buffer A. Membrane spectra of the purified membranes was carried out as previously reported with slight modifications. ${ }^{17,30}$ For the spectral analysis, the oxidised spectrum from 400 to $700 \mathrm{~nm}$ was recorded on a Cytation 3 (BioTek) plate reader upon addition of $100 \mu \mathrm{M}$ potassium ferricyanide. The reduced spectra were recorded after $6 \mathrm{~min}$ incubation with $10 \mathrm{mM}$ of reduced nicotinamide adenine dinucleotide (NADH) as a reducing agent. The data were analysed as difference between reduced and oxidised spectra and the baseline was adjusted to present the spectra in the same range of values. The graphs represent data on the range from 500 to $700 \mathrm{~nm}$ as a focus area. For proton translocation experiment, the quenching of the fluorescent ACMA dye was recorded upon addition of $0.5 \mathrm{mM}$ NADH using Cary Eclipse Fluorescence spectrophotometer (Varian Inc.) according to Hotra et al. ${ }^{41}$

\section{ANXILLARY INFORMATION}

Supporting information: Figure S1, Sequence alignment of subunit CydA of cyt- $b d$; Figure S2, Sequence alignment of subunit CydB of cyt- $b d$; Figure S3, Experimental verification of the assays developed for mutagenesis studies; Figure S4, Comparison of effects of two deletions in CydA; Figure S5, Heme content analysis in WT BCG and BCG $\triangle c y d A B:$ :pMV306-cydABDC; Figure S6, Functional evaluation of mutant cyt- $b d$ expressed from the high-copy vector 
pMV262; Figure S7, Growth rate in 7H9 broth media; Figure S8, Growth rate in defined media supplemented with $20 \mathrm{mM}$ acetate.

Corresponding Author Information: Prof. Dr. Gerhard Grüber, Tel.: + 65 - 6316 2989, E-mail: ggrueber@ntu.edu.sg_and Prof. Dr. Kevin Pethe, Tel.: + 65 - 6592 3958, E-mail: kevin.pethe@,ntu.edu.sg

Present Author Addresses: ${ }^{1}$ Nanyang Technological University, School of Biological Sciences, 60 Nanyang Drive, Singapore 637551, Republic of Singapore, ${ }^{2}$ Lee Kong Chian School of Medicine, 59 Nanyang Drive, Singapore 636921, Republic of Singapore.

Author contributions: E.S., M.S.S.M., G.G., and K.P. conceived and designed the experiments. E.S. and M.S.S.M. performed the experiments and analyzed the data. E.S., M.S.S.M., G.G., and K.P. wrote the paper. ${ }^{\#}$ These authors have equal contribution

Acknowledgements: We thank Lee Bei Shi for discussions and experimental assistance during the course of the project and Chong Shi Min Sherilyn for optimization of membrane isolation and spectral analysis assays. This research and the $\mathrm{PhD}$ scholarship of Ekaterina Sviriaeva were supported by the National Research Foundation (NRF) Singapore, NRF Competitive Research Programme (CRP), Grant Award Number NRF-CRP18-2017-01 to G.G. and K.P.

\section{References:}

1. Rao, S. P.; Alonso, S.; Rand, L.; Dick, T.; Pethe, K., The protonmotive force is required for maintaining ATP homeostasis and viability of hypoxic, nonreplicating Mycobacterium tuberculosis. Proc Natl Acad Sci U S A 2008, 105 (33), 11945-50. DOI:

10.1073/pnas.0711697105.

2. Koul, A.; Vranckx, L.; Dendouga, N.; Balemans, W.; Van den Wyngaert, I.; Vergauwen, K.; Gohlmann, H. W.; Willebrords, R.; Poncelet, A.; Guillemont, J.; Bald, D.; Andries, K., Diarylquinolines are bactericidal for dormant mycobacteria as a result of disturbed ATP homeostasis. J Biol Chem 2008, 283 (37), 25273-80. DOI: 10.1074/jbc.M803899200.

3. Cook, G. M.; Hards, K.; Dunn, E.; Heikal, A.; Nakatani, Y.; Greening, C.; Crick, D. C.; Fontes, F. L.; Pethe, K.; Hasenoehrl, E.; Berney, M., Oxidative Phosphorylation as a Target Space for Tuberculosis: Success, Caution, and Future Directions. Microbiol Spectr 2017, 5 (3). DOI: 10.1128/microbiolspec.TBTB2-0014-2016.

4. $\quad$ Arora, K.; Ochoa-Montano, B.; Tsang, P. S.; Blundell, T. L.; Dawes, S. S.; Mizrahi, V.; Bayliss, T.; Mackenzie, C. J.; Cleghorn, L. A.; Ray, P. C.; Wyatt, P. G.; Uh, E.; Lee, J.; Barry, C. E., 3rd; Boshoff, H. I., Respiratory flexibility in response to inhibition of cytochrome C oxidase in Mycobacterium tuberculosis. Antimicrob Agents Chemother 2014, 58 (11), 6962-5. DOI: 10.1128/AAC.03486-14.

5. $\quad$ Pethe, K.; Bifani, P.; Jang, J.; Kang, S.; Park, S.; Ahn, S.; Jiricek, J.; Jung, J.; Jeon, H. K.; Cechetto, J.; Christophe, T.; Lee, H.; Kempf, M.; Jackson, M.; Lenaerts, A. J.; Pham, H.; Jones, V.; Seo, M. J.; Kim, Y. M.; Seo, M.; Seo, J. J.; Park, D.; Ko, Y.; Choi, I.; Kim, R.; Kim, S. Y.; Lim, S.; Yim, S. A.; Nam, J.; Kang, H.; Kwon, H.; Oh, C. T.; Cho, Y.; Jang, Y.; Kim, J.; Chua, 
A.; Tan, B. H.; Nanjundappa, M. B.; Rao, S. P.; Barnes, W. S.; Wintjens, R.; Walker, J. R.; Alonso, S.; Lee, S.; Kim, J.; Oh, S.; Oh, T.; Nehrbass, U.; Han, S. J.; No, Z.; Lee, J.; Brodin, P.; Cho, S. N.; Nam, K.; Kim, J., Discovery of Q203, a potent clinical candidate for the treatment of tuberculosis. Nat Med 2013, 19 (9), 1157-60. DOI: 10.1038/nm.3262.

6. Rybniker, J.; Vocat, A.; Sala, C.; Busso, P.; Pojer, F.; Benjak, A.; Cole, S. T., Lansoprazole is an antituberculous prodrug targeting cytochrome bc1. Nat Commun 2015, 6, 7659. DOI: $10.1038 /$ ncomms 8659.

7. Phummarin, N.; Boshoff, H. I.; Tsang, P. S.; Dalton, J.; Wiles, S.; Barry Rd, C. E.; Copp, B. R., SAR and identification of 2-(quinolin-4-yloxy)acetamides as Mycobacterium tuberculosis cytochrome bc1 inhibitors. Medchemcomm 2016, 7 (11), 2122-2127. DOI: 10.1039/c6md00236f. 8. Chandrasekera, N. S.; Berube, B. J.; Shetye, G.; Chettiar, S.; O'Malley, T.; Manning, A.; Flint, L.; Awasthi, D.; Ioerger, T. R.; Sacchettini, J.; Masquelin, T.; Hipskind, P. A.; Odingo, J.; Parish, T., Improved Phenoxyalkylbenzimidazoles with Activity against Mycobacterium tuberculosis Appear to Target QcrB. ACS Infect Dis 2017, 3 (12), 898-916. DOI: 10.1021/acsinfecdis.7b00112.

9. $\quad$ Scherr, N.; Bieri, R.; Thomas, S. S.; Chauffour, A.; Kalia, N. P.; Schneide, P.; Ruf, M. T.; Lamelas, A.; Manimekalai, M. S. S.; Gruber, G.; Ishii, N.; Suzuki, K.; Tanner, M.; Moraski, G. C.; Miller, M. J.; Witschel, M.; Jarlier, V.; Pluschke, G.; Pethe, K., Targeting the Mycobacterium ulcerans cytochrome bc1:aa3 for the treatment of Buruli ulcer. Nat Commun 2018, 9 (1), 5370. DOI: 10.1038/s41467-018-07804-8.

10. Liu, Y.; Gao, Y.; Liu, J.; Tan, Y.; Liu, Z.; Chhotaray, C.; Jiang, H.; Lu, Z.; Chiwala, G.; Wang, S.; Makafe, G.; Islam, M. M.; Hameed, H. M. A.; Cai, X.; Wang, C.; Li, X.; Tan, S.; Zhang, T., The compound TB47 is highly bactericidal against Mycobacterium ulcerans in a Buruli ulcer mouse model. Nat Commun 2019, 10 (1), 524. DOI: 10.1038/s41467-019-08464-y. 11. Kalia, N. P.; Hasenoehrl, E. J.; Ab Rahman, N. B.; Koh, V. H.; Ang, M. L. T.; Sajorda, D. R.; Hards, K.; Gruber, G.; Alonso, S.; Cook, G. M.; Berney, M.; Pethe, K., Exploiting the synthetic lethality between terminal respiratory oxidases to kill Mycobacterium tuberculosis and clear host infection. Proc Natl Acad Sci U S A 2017, 114 (28), 7426-7431. DOI:

10.1073/pnas.1706139114.

12. Lamprecht, D. A.; Finin, P. M.; Rahman, M. A.; Cumming, B. M.; Russell, S. L.; Jonnala, S. R.; Adamson, J. H.; Steyn, A. J., Turning the respiratory flexibility of Mycobacterium tuberculosis against itself. Nat Commun 2016, 7, 12393. DOI: 10.1038/ncomms12393.

13. Hards, K.; Robson, J. R.; Berney, M.; Shaw, L.; Bald, D.; Koul, A.; Andries, K.; Cook, G. M., Bactericidal mode of action of bedaquiline. J Antimicrob Chemother 2015, 70 (7), 202837. DOI: $10.1093 / \mathrm{jac} / \mathrm{dkv} 054$.

14. Lu, P.; Heineke, M. H.; Koul, A.; Andries, K.; Cook, G. M.; Lill, H.; van Spanning, R.; Bald, D., The cytochrome bd-type quinol oxidase is important for survival of Mycobacterium smegmatis under peroxide and antibiotic-induced stress. Sci Rep 2015, 5, 10333. DOI: 10.1038/srep10333.

15. Berney, M.; Hartman, T. E.; Jacobs, W. R., Jr., A Mycobacterium tuberculosis cytochrome bd oxidase mutant is hypersensitive to bedaquiline. MBio 2014, 5 (4), e01275-14. DOI: $10.1128 / \mathrm{mBio} .01275-14$.

16. Cruz-Ramos, H.; Cook, G. M.; Wu, G.; Cleeter, M. W.; Poole, R. K., Membrane topology and mutational analysis of Escherichia coli CydDC, an ABC-type cysteine exporter 
required for cytochrome assembly. Microbiology 2004, 150 (Pt 10), 3415-27. DOI: 10.1099/mic.0.27191-0.

17. Kana, B. D.; Weinstein, E. A.; Avarbock, D.; Dawes, S. S.; Rubin, H.; Mizrahi, V., Characterization of the cydAB-encoded cytochrome bd oxidase from Mycobacterium smegmatis. J Bacteriol 2001, 183 (24), 7076-86. DOI: 10.1128/JB.183.24.7076-7086.2001.

18. Borisov, V. B.; Forte, E.; Davletshin, A.; Mastronicola, D.; Sarti, P.; Giuffre, A., Cytochrome bd oxidase from Escherichia coli displays high catalase activity: an additional defense against oxidative stress. FEBS Lett 2013, 587 (14), 2214-8. DOI:

10.1016/j.febslet.2013.05.047.

19. Belevich, I.; Borisov, V. B.; Zhang, J.; Yang, K.; Konstantinov, A. A.; Gennis, R. B.; Verkhovsky, M. I., Time-resolved electrometric and optical studies on cytochrome bd suggest a mechanism of electron-proton coupling in the di-heme active site. Proc Natl Acad Sci US A 2005, 102 (10), 3657-62. DOI: 10.1073/pnas.0405683102.

20. Al-Attar, S.; Yu, Y.; Pinkse, M.; Hoeser, J.; Friedrich, T.; Bald, D.; de Vries, S., Cytochrome bd Displays Significant Quinol Peroxidase Activity. Sci Rep 2016, 6, 27631. DOI: 10.1038/srep27631.

21. Murali, R.; Gennis, R. B., Functional importance of Glutamate-445 and Glutamate-99 in proton-coupled electron transfer during oxygen reduction by cytochrome bd from Escherichia coli. Biochim Biophys Acta Bioenerg 2018, 1859 (8), 577-590. DOI:

10.1016/j.bbabio.2018.04.012.

22. Mogi, T.; Akimoto, S.; Endou, S.; Watanabe-Nakayama, T.; Mizuochi-Asai, E.; Miyoshi, H., Probing the ubiquinol-binding site in cytochrome bd by site-directed mutagenesis. Biochemistry 2006, 45 (25), 7924-30. DOI: 10.1021/bi060192w.

23. Safarian, S.; Rajendran, C.; Muller, H.; Preu, J.; Langer, J. D.; Ovchinnikov, S.; Hirose, T.; Kusumoto, T.; Sakamoto, J.; Michel, H., Structure of a bd oxidase indicates similar mechanisms for membrane-integrated oxygen reductases. Science 2016, 352 (6285), 583-6. DOI: 10.1126/science.aaf2477.

24. Safarian, S.; Hahn, A.; Mills, D. J.; Radloff, M.; Eisinger, M. L.; Nikolaev, A.; MeierCredo, J.; Melin, F.; Miyoshi, H.; Gennis, R. B.; Sakamoto, J.; Langer, J. D.; Hellwig, P.; Kuhlbrandt, W.; Michel, H., Active site rearrangement and structural divergence in prokaryotic respiratory oxidases. Science 2019, 366 (6461), 100-104. DOI: 10.1126/science.aay0967.

25. Borisov, V. B.; Gennis, R. B.; Hemp, J.; Verkhovsky, M. I., The cytochrome bd respiratory oxygen reductases. Biochim Biophys Acta 2011, 1807 (11), 1398-413. DOI: 10.1016/j.bbabio.2011.06.016.

26. Hahn, A.; Vonck, J.; Mills, D. J.; Meier, T.; Kuhlbrandt, W., Structure, mechanism, and regulation of the chloroplast ATP synthase. Science 2018, 360 (6389). DOI:

10.1126/science.aat4318.

27. Andries, K.; Verhasselt, P.; Guillemont, J.; Gohlmann, H. W.; Neefs, J. M.; Winkler, H.; Van Gestel, J.; Timmerman, P.; Zhu, M.; Lee, E.; Williams, P.; de Chaffoy, D.; Huitric, E.; Hoffner, S.; Cambau, E.; Truffot-Pernot, C.; Lounis, N.; Jarlier, V., A diarylquinoline drug active on the ATP synthase of Mycobacterium tuberculosis. Science 2005, 307 (5707), 223-7. DOI: $10.1126 /$ science. 1106753 .

28. Thesseling, A.; Burschel, S.; Wohlwend, D.; Friedrich, T., The long Q-loop of Escherichia coli cytochrome bd oxidase is required for assembly and structural integrity. FEBS Lett 2020. DOI: 10.1002/1873-3468.13749. 
29. Thesseling, A.; Rasmussen, T.; Burschel, S.; Wohlwend, D.; Kagi, J.; Muller, R.; Bottcher, B.; Friedrich, T., Homologous bd oxidases share the same architecture but differ in mechanism. Nat Commun 2019, 10 (1), 5138. DOI: 10.1038/s41467-019-13122-4.

30. Small, J. L.; Park, S. W.; Kana, B. D.; Ioerger, T. R.; Sacchettini, J. C.; Ehrt, S., Perturbation of cytochrome c maturation reveals adaptability of the respiratory chain in Mycobacterium tuberculosis. MBio 2013, 4 (5), e00475-13. DOI: 10.1128/mBio.00475-13.

31. Diacon, A. H.; Pym, A.; Grobusch, M. P.; de los Rios, J. M.; Gotuzzo, E.; Vasilyeva, I.; Leimane, V.; Andries, K.; Bakare, N.; De Marez, T.; Haxaire-Theeuwes, M.; Lounis, N.; Meyvisch, P.; De Paepe, E.; van Heeswijk, R. P.; Dannemann, B.; Group, T. C. S., Multidrugresistant tuberculosis and culture conversion with bedaquiline. $N$ Engl J Med 2014, 371 (8), 723 32. DOI: 10.1056/NEJMoa1313865.

32. Pym, A. S.; Diacon, A. H.; Tang, S. J.; Conradie, F.; Danilovits, M.; Chuchottaworn, C.; Vasilyeva, I.; Andries, K.; Bakare, N.; De Marez, T.; Haxaire-Theeuwes, M.; Lounis, N.; Meyvisch, P.; Van Baelen, B.; van Heeswijk, R. P.; Dannemann, B.; Group, T. C. S., Bedaquiline in the treatment of multidrug- and extensively drug-resistant tuberculosis. Eur Respir J 2016, 47 (2), 564-74. DOI: 10.1183/13993003.00724-2015.

33. Swindells, S., New drugs to treat tuberculosis. F1000 Med Rep 2012, 4, 12. DOI: 10.3410/M4-12.

34. Goojani, H. G.; Konings, J.; Hakvoort, H.; Hong, S.; Gennis, R. B.; Sakamoto, J.; Lill, H.; Bald, D., The carboxy-terminal insert in the Q-loop is needed for functionality of Escherichia coli cytochrome bd-I. Biochim Biophys Acta Bioenerg 2020, 148175. DOI:

10.1016/j.bbabio.2020.148175.

35. Yang, K.; Zhang, J.; Vakkasoglu, A. S.; Hielscher, R.; Osborne, J. P.; Hemp, J.; Miyoshi, H.; Hellwig, P.; Gennis, R. B., Glutamate 107 in subunit I of the cytochrome bd quinol oxidase from Escherichia coli is protonated and near the heme $\mathrm{d} /$ heme b595 binuclear center.

Biochemistry 2007, 46 (11), 3270-8. DOI: 10.1021/bi061946+.

36. McWilliam, H.; Li, W.; Uludag, M.; Squizzato, S.; Park, Y. M.; Buso, N.; Cowley, A. P.; Lopez, R., Analysis Tool Web Services from the EMBL-EBI. Nucleic Acids Res 2013, 41 (Web Server issue), W597-600. DOI: 10.1093/nar/gkt376.

37. Robert, X.; Gouet, P., Deciphering key features in protein structures with the new ENDscript server. Nucleic Acids Res 2014, 42 (Web Server issue), W320-4. DOI: 10.1093/nar/gku316.

38. Waterhouse, A.; Bertoni, M.; Bienert, S.; Studer, G.; Tauriello, G.; Gumienny, R.; Heer, F. T.; de Beer, T. A. P.; Rempfer, C.; Bordoli, L.; Lepore, R.; Schwede, T., SWISS-MODEL: homology modelling of protein structures and complexes. Nucleic Acids Res 2018, 46 (W1), W296-W303. DOI: 10.1093/nar/gky427.

39. Camacho, C.; Coulouris, G.; Avagyan, V.; Ma, N.; Papadopoulos, J.; Bealer, K.; Madden, T. L., BLAST+: architecture and applications. BMC Bioinformatics 2009, 10, 421. DOI: $10.1186 / 1471-2105-10-421$.

40. Remmert, M.; Biegert, A.; Hauser, A.; Soding, J., HHblits: lightning-fast iterative protein sequence searching by HMM-HMM alignment. Nat Methods 2011, 9 (2), 173-5. DOI: 10.1038/nmeth.1818.

41. Hotra, A.; Suter, M.; Biuković, G.; Ragunathan, P.; Kundu, S.; Dick, T.; Grüber, G., Deletion of a unique loop in the mycobacterial F-ATP synthase gamma subunit sheds light on its inhibitory role in ATP hydrolysis-driven $\mathrm{H}(+)$ pumping. FEBS J 2016, 283 (10), 1947-61. DOI: $10.1111 /$ febs. 13715 . 

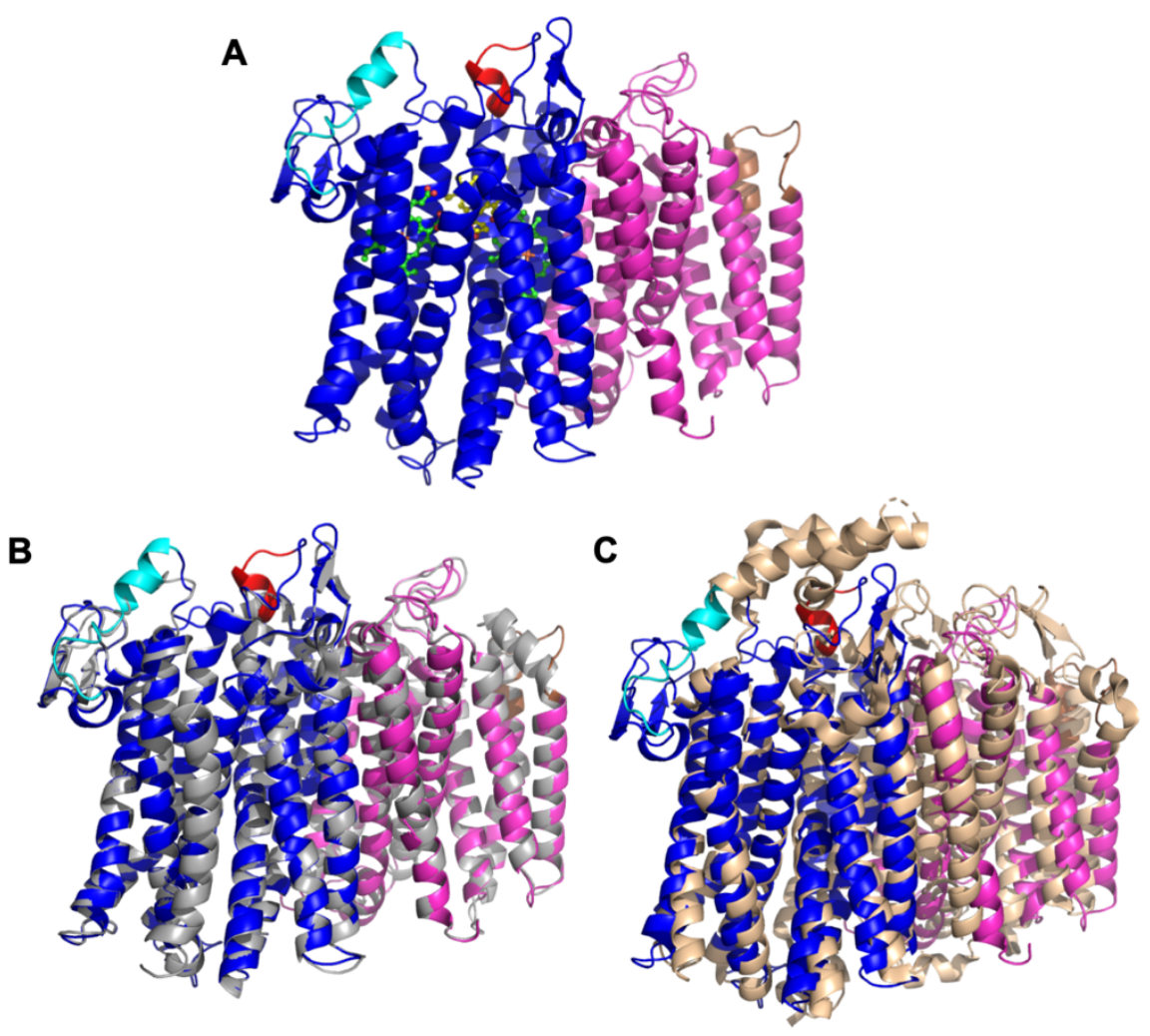

Fig. 1. A, Cartoon representation of the homology model of cyt- $b d$ from $M$. tuberculosis. Subunit CydA is highlighted in blue and subunit $\mathrm{CydB}$ in magenta. In subunit $\mathrm{CydA}$, the unique short region ${ }_{412}$ QLVRLTVKA $_{420}$ is highlighted in red and the region ${ }_{307}$ SGVTLQGIRDLQQEYQQ $_{323}$ is shown as cyan. In subunit $\mathrm{CydB}$, the shorter region in the mycobacterium species is shown in brown. The heme groups are shown in ball and stick representation displaying heme $b$ in green and heme $d$ in yellow. Structural superposition of the M. tuberculosis model of cyt- $b d$ with $(B) G$. thermodenitrificans crystal structure (grey; PDB ID: 5DOQ) and (C) cryo-EM structure of $E$. coli (sand; PDB ID: 6RKO). 

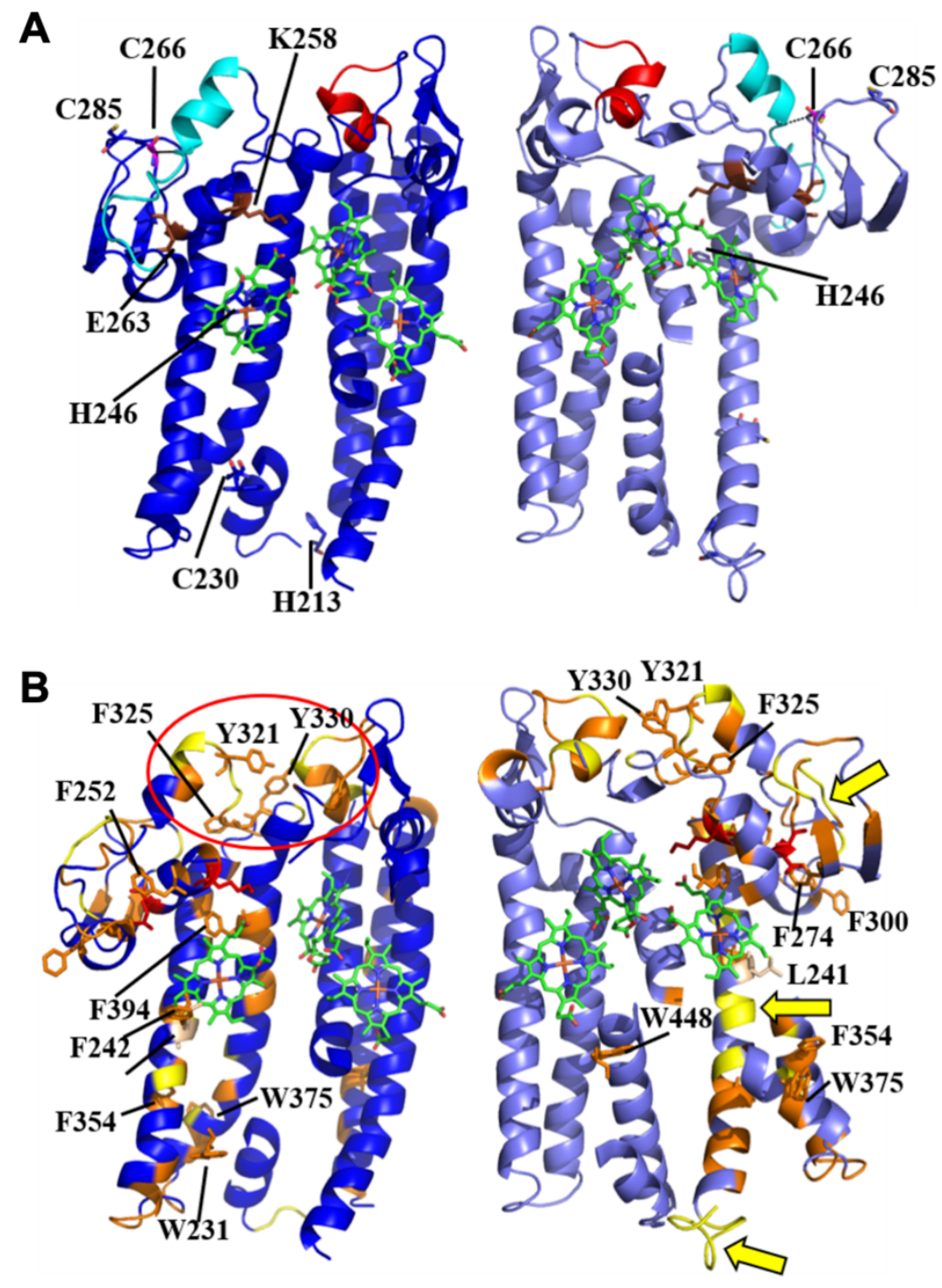

Fig. 2. $A$, Center cut open view of subunit CydA, as a book outlook, with the cut open side in light shade of blue showing the cystine and histidine residues. The proposed menaquinol binding residues (K258 and E263) are shown in brown. B, Cut open view of subunit CydA showing the aromatic residues. The four $\mathrm{C}$-terminal helices 10-13 show variations of residues for the mycobacterium (orange and yellow region). The residue difference for the non-pathogenic $M$. smegmatis is shown in yellow and the regions marked by yellow arrow. An aromatic cluster near the proposed menaquinol binding region (Y321, Y330 and F325) and the unique short region ${ }_{412} \mathrm{QLVRLTVKA}_{420}$ is circled. The heme groups are shown in green stick representation. 


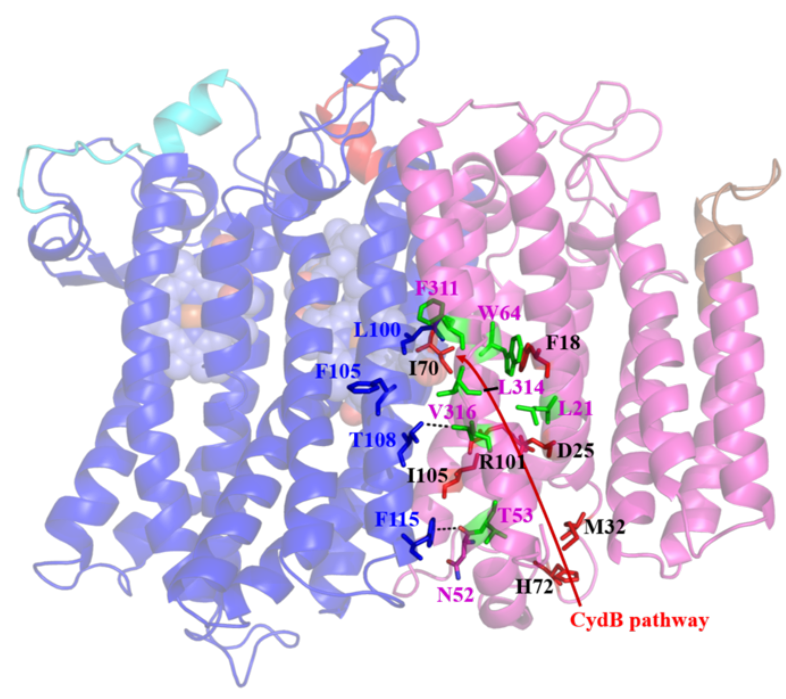

Fig. 3. Cartoon representation of the M. tuberculosis cytochrome $b d$ oxidase model illustrating the CydB pathway's residues highlighted in red (H37, M32, I105, D25, R101, F18, and I70), and the proton transfer pathway is shown by red arrow. The generated mutant residues closer to the CydB pathways residues are shown in green. Subunit CydA and its residues are highlighted and labelled in blue, while subunit $\mathrm{CydB}$ and the critical residues are shown in magenta. In subunit CydA, the unique short region ${ }_{412} \mathrm{QLVRLTVKA}_{420}$ is shown in red and the region ${ }_{307}$ SGVTLQGIRDLQQEYQQ 323 is displayed in cyan. In subunit CydB, the shorter region in the mycobacteria is shown in brown. The heme groups are revealed in sphere representation. 
A

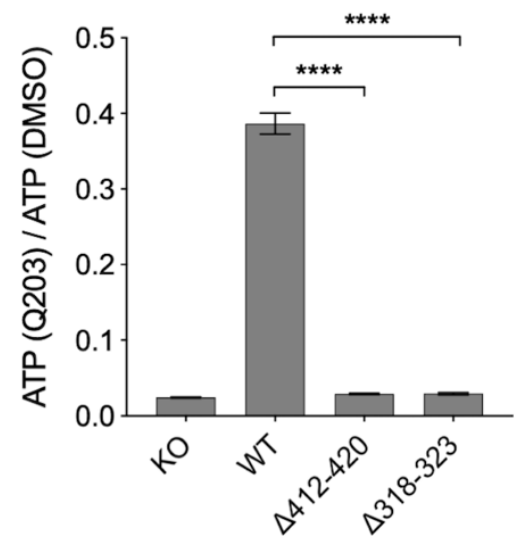

B

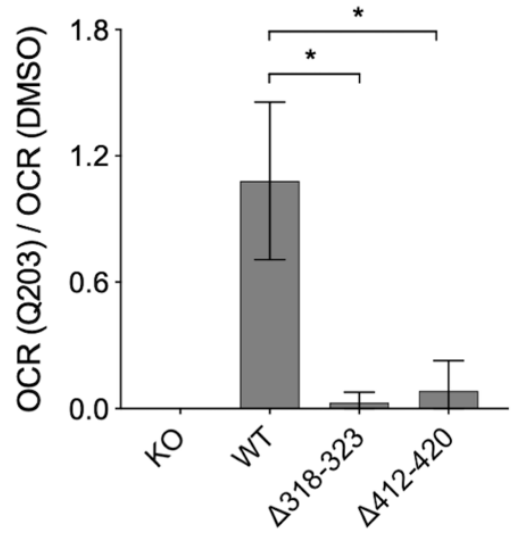

C

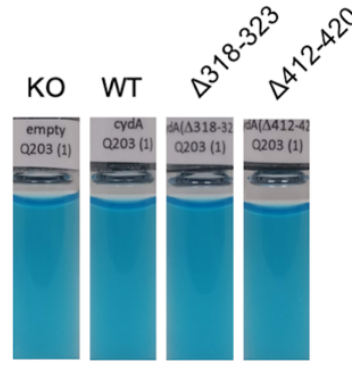

$120 \mathrm{~h}$

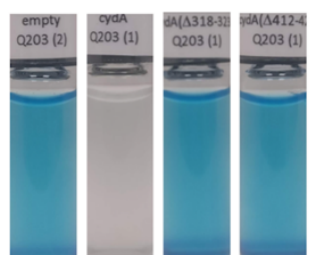

Fig. 4. Activity of cyt- $b d$ is significantly reduced in mutant cyt- $b d$ with $\mathrm{CydA}_{\triangle 318-323}$ and CydA $_{\triangle 412-420}$ deletions. $A$, ATP levels are drastically reduced in strains carrying deletion mutants. $B$, OCR ratio quantification in strains with mutant cyt-bd. $C$, Methylene blue assay supports results of OCR ratio quantification. *: $\mathrm{P}<0.05, * * *:<\mathrm{P} 0.001, * * * *: \mathrm{P}<0.0001$, multiple comparison with WT by one-way ANOVA test. All experiments were performed in triplicates and repeated at least twice, methylene blue assay was performed in duplicates and repeated at least twice. 
A

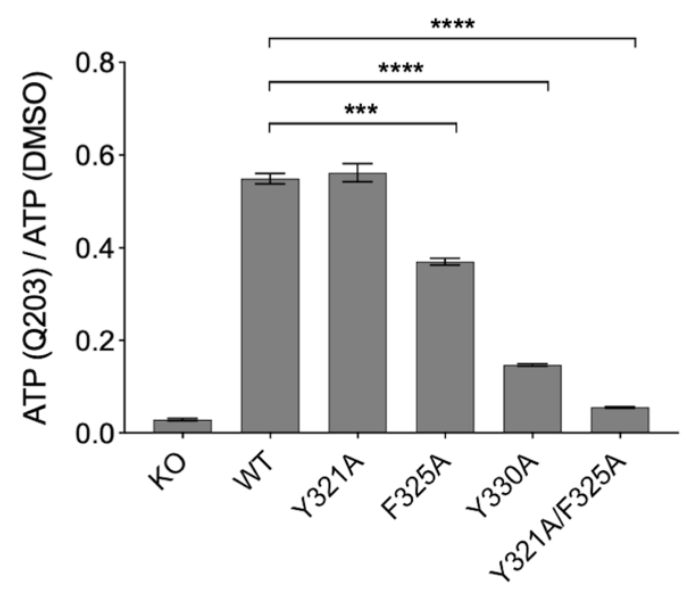

B
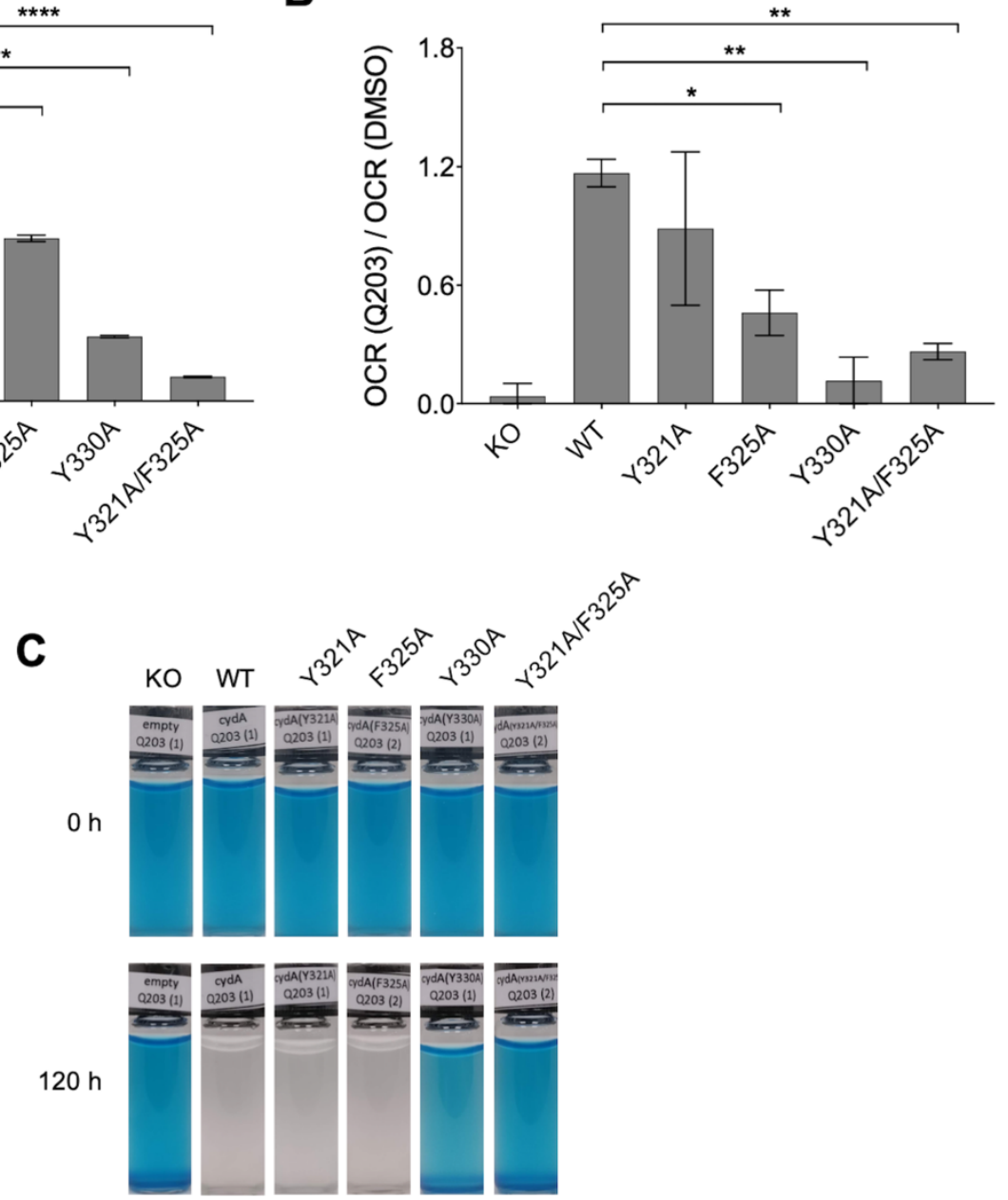

Fig. 5. Activity of cyt- $b d$ with amino acid substitutions in subunit CydA. $A, B$, ATP production level and OCR ratio, respectively, are significantly impaired in CydA Y330A and CydA Y321A/F325A double mutant but not in CydA Y321A and CydA $\mathrm{F}_{325 \mathrm{~A}}$ mutants. $\mathrm{C}$, Methylene blue assay supports OCR ratio quantification data. $*: \mathrm{P}<0.05, * *: \mathrm{P}<0.01, * * *:<\mathrm{P} 0.001, * * * *: \mathrm{P}<0.0001$, multiple comparison with WT by one-way ANOVA test. All experiments were performed in triplicates and repeated at least twice, methylene blue assay was performed in duplicates and repeated at least twice. 
A

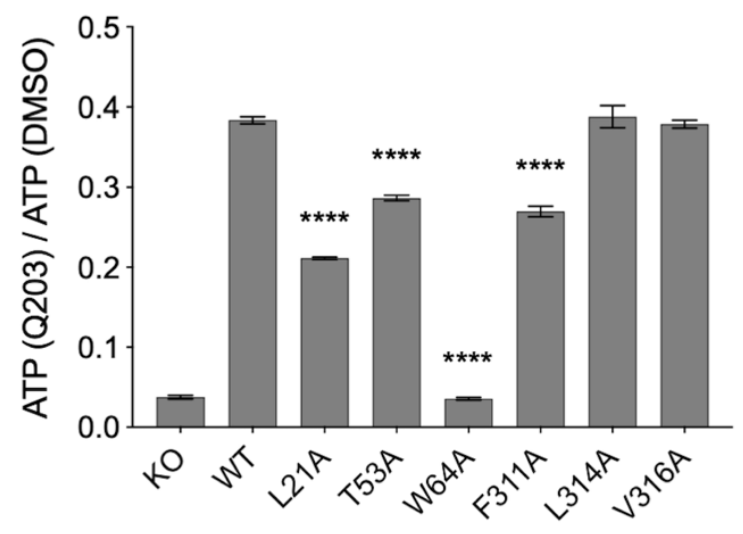

B

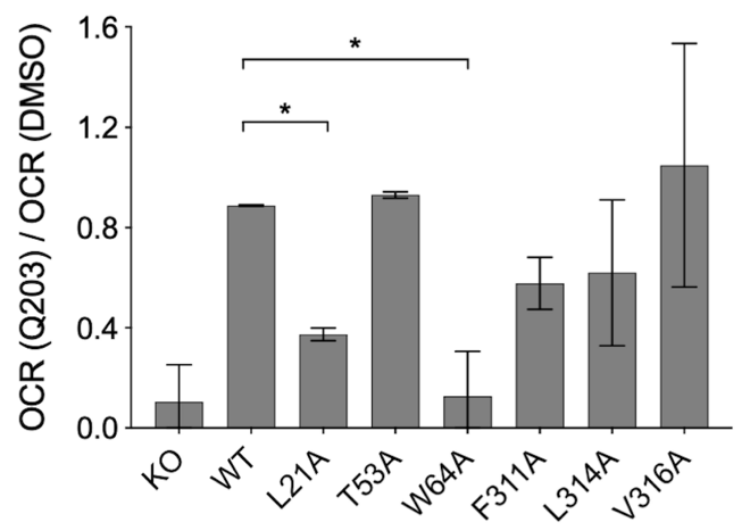

C

$\mathrm{Oh}$

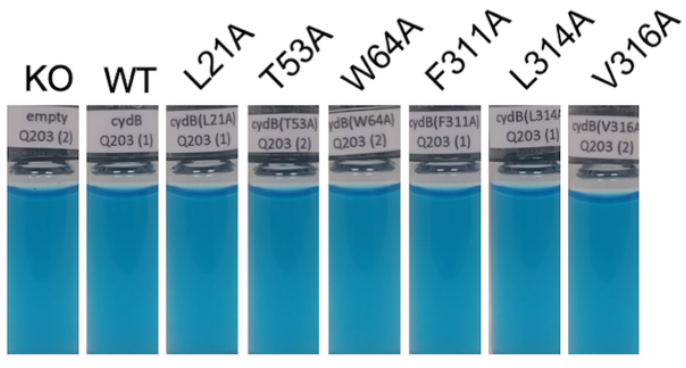

$120 \mathrm{~h}$

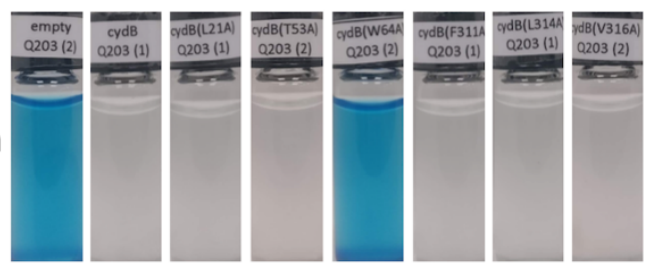

Fig. 6. Activity of cyt- $b d$ carrying mutations in subunit CydB. $A$, ATP production level is significantly impaired in mutant cyt- $b d$ with $\mathrm{CydB}_{\mathrm{L2} 2 \mathrm{~A}}, \mathrm{CydB}_{\mathrm{T} 53 \mathrm{~A}}, \mathrm{CydB}_{\mathrm{W} 64 \mathrm{~A}}$, and $\mathrm{CydB}_{\mathrm{F} 311 \mathrm{~A}} . B$, OCR ratio is significantly reduced in $\mathrm{CydB}_{\mathrm{L} 21 \mathrm{~A}}$, and $\mathrm{CydB}_{\mathrm{W} 64 \mathrm{~A}}$ mutants. $C$. Methylene blue assay results support data from OCR quantification assay. *: $\mathrm{P}<0.05, * *: \mathrm{P}<0.01, * * * *: \mathrm{P}<0.0001$, multiple comparison with WT by one-way ANOVA test. All experiments in triplicates and repeated at least twice, methylene blue assay was performed in duplicates and repeated at least twice. 


\section{A}

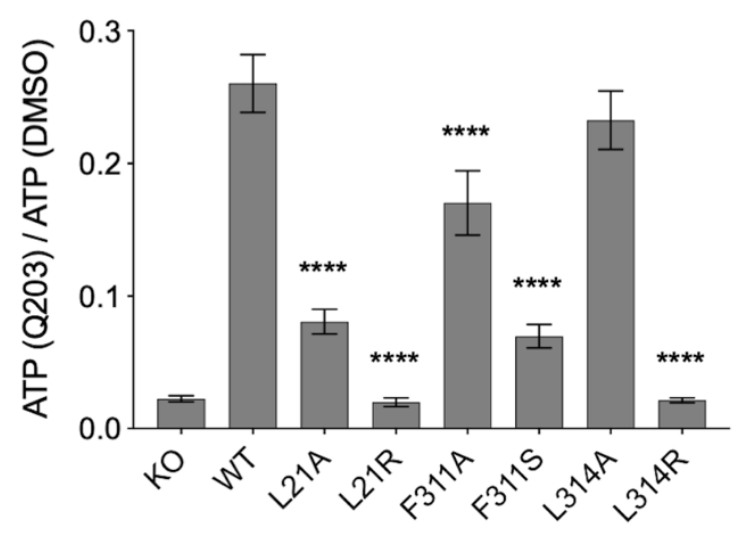

B

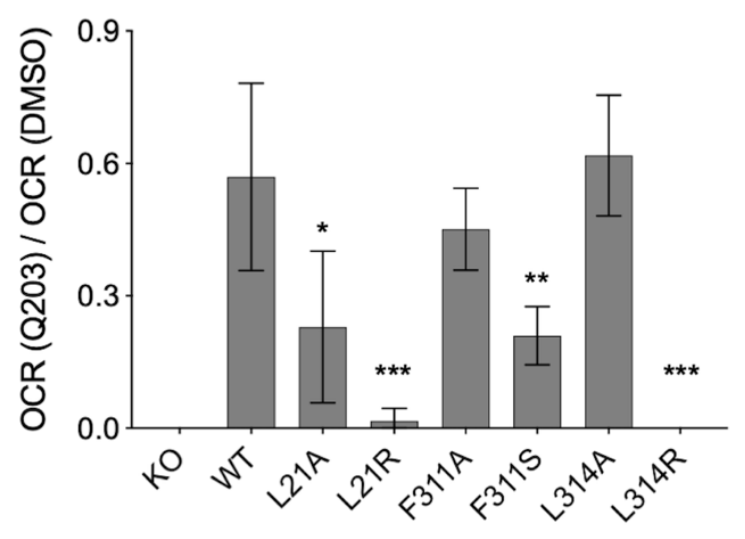

Fig. 7. Substitution of L21, F311 and L314 with charged residues affects functioning of the complex to further extent then substitution with neutral alanine. $A, B, C$, Comparison of ATP production upon treatment with Q203 in mutants. $D, E, F$, Comparison of oxygen production upon treatment with Q203 in mutants. *: $\mathrm{P}<0.05, * *: \mathrm{P}<0.01,{ }^{* * *}: \mathrm{P}<0.001, * * * *: \mathrm{P}<0.0001$, multiple comparison with WT by one-way ANOVA test. All experiments were performed in triplicates and repeated at least twice. 

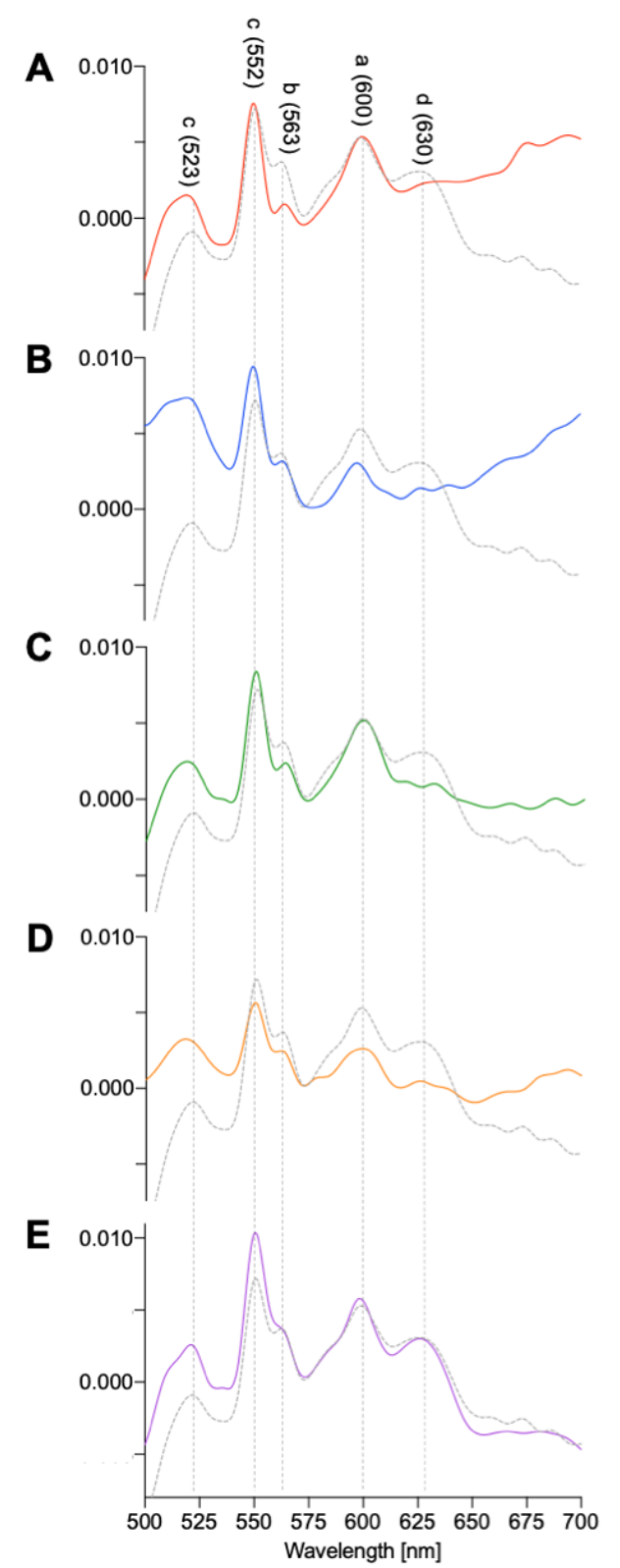

Fig. 8. Influence of the mutations in $\mathrm{CydA}$ and $\mathrm{CydB}$ on cyt- $b d$ integrity. The heme $d$-specific peak at $630 \mathrm{~nm}$ is significantly reduced in $\mathrm{CydA}_{\triangle 318-323}(\mathrm{~A}), \mathrm{CydA}_{\triangle 412-420}(\mathrm{~B}), \mathrm{CydA}_{\mathrm{y} 330 \mathrm{~A}}(\mathrm{C})$, $\mathrm{CydB}_{\mathrm{W} 64 \mathrm{~A}}(\mathrm{D})$, but present in the $\mathrm{CydB}_{\mathrm{L} 314 \mathrm{~A}}$ mutant (E). NADH-reduced minus potassium ferricyanide-oxidized spectra were obtained from membrane preparations of M. bovis BCG $\triangle c y d A B:$ :pMV262-cydABDC WT or carrying the specified mutations. The spectrum for M. bovis BCG $\triangle c y d A B:: p M V 262-c y d A B D C$ WT is presented in gray dashed line on each graph. Specific peaks for hemes $c(523 \mathrm{~nm}, 552 \mathrm{~nm}), b(563 \mathrm{~nm}), a(600 \mathrm{~nm})$ and $d(630 \mathrm{~nm})$ are labeled on the graph. The spectra are representative of three independent experiments. 


\section{For Table of Contents Only}

Manuscript title: Novel features and functional importance of key residues of the Mycobacterium tuberculosis cytochrome $b d$

Authors: Ekaterina Sviriaeva ${ }^{1,2 \#}$, Malathy Sony Subramanian Manimekalai 1,\#, Gerhard Grüber ${ }^{1, *}$ and Kevin Pethe ${ }^{1,2, *}$

Brief synopsis: The 3D model of mycobacterial cyt- $b d$ was generated based on a published structure of of the Geobacillus thermodenitrificans cyt-bd. Together with it and protein sequence alignment key features of mycobacterial cyt- $b d$ were identified and their significance was confirmed in model organism using whole-cell bacteria assays and spectral analysis of the membranes.

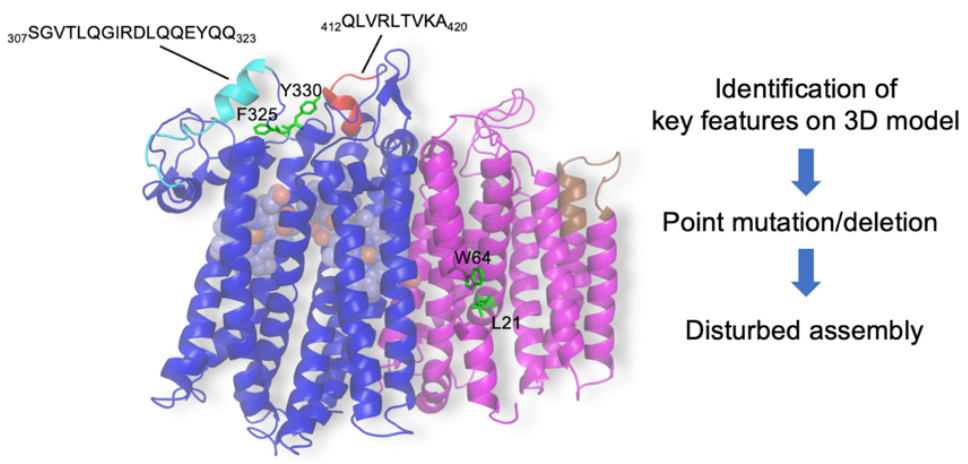

